the ends of these issuing from the respective angles of the wound. The wound in the neck was then closed, and here I may say that this was a mistake, as it suppurated later and had to be completely undone; no harm, however, ensued.

By this complete operation I succeeded in removing nearly the whole of the septic clot and in addition cut off the main supply of septic material from entering the general circulation. I therefore looked for a cessation of the symptoms which had previously existed and hoped for an uninterrupted recovery. But in this I was disappointed. The operation was performed on Oct 19th and without giving the detailed notes taken from day to day suffice it to say that his symptoms continued. He had repeated rigors and high temperatures, a chart of which is appended. In the intervals of the rigors he felt fairly well but extremely weak.

On Oct. 25th my note says: "Breathing seems a little laboured; crepitations to be heard at the third left rib in front and impaired resonance at the left base." The physical signs remained thus for the next day or two and the rigors were repeated. I therefore resolved to adopt a method which $I$ have not seen described as having been employed for the treatment of undoubted general septic infection.

I think there can be no doubt that it was a case of true septic infection as distinct from septic intoxication-i.e., septicæmia as against sapræmia-this view being supported by the fact that the main part, if not the entire supply, of septic material had been cut off, in spite of which the constitutional symptoms persisted and the condition in the lungs above described supervened. His only hope of recovery I considered lay in an increase in his power of eliminating the poison with which he was saturated and this I thought I could effect for him by largeiy diluting his blood. On Oct. 30th I therefore admitted into his right median basilic vein three pints of normal saline solution and one ounce of brandy. On Nov. 2nd my note says: "Has had no rigor for several days and there has certainly been an improvement in his condition ever since the transfusion." (See temperature chart.) "There are some dulness at the left base and a diminution of breath sounds and vocal resonance." On the 3rd I opened the median basilic vein of the left arm and removed nearly one pint of blood; I then transfused him with another three pints of normal saline solution and one ounce of brandy. On the 10th my note states: "He has improved immensely since the last transfusion. For the last three days his temperature has been practically normal." (See temperature chart.) On the 3rd the second transfusion took place and the temperature did not finally reach normal until the 7 th, but in the intervening four days it was lower on the average and the elevations were not so great when they did occur. The chart for these days suggests a final flicker of the disease. The quantity of urine excreted subsequently to the transfusions did not show any great increase, though it was a little more, but there was marked sweating, his garments being frequently changed when wet through. It was in this way, possibly, that he eliminated toxic elements.

His final recovery was now uninterrupted and he is at the present time perfectly well and was shown at a meeting of the Nottingham Medico.Chirurgical Society on Jan. 21st, when this account of the case was read.

In conclusion, I would say that this short paper does not presume to be an elaborate account of the treatment of chronic suppuration in the middle ear, but merely expresses the opinion that that disease is eminently curable, and in view of its complications, should be cured. Secondly, in a brief and imperfect manner the lines on which such treatment should be carried out are suggested and outlined. Finally, in respect of the thrombosed lateral sinus, the operation described is in no way novel, but is the method very frequently adopted. In regard to the history of the case, however, subsequent to the operation, the evidence which the case affords seems to me to warrant the opinion that the patient's life was saved, when he would otherwise have died from septicæmia, by the transfusions which were performed and 1 venture to submit this for publication as I have not seen any similar case recorded.

Nottingham.

New Workhouse INFIRMary at BideFord.The new workhouse infirmary at Bideford, Devon, was formally opened on June 2nd in the presence of a large gathering. The building will accommodate 38 persons and has been erected at a cost of $£ 3248$.

\section{THE ANILINE DYES AS THERAPEUTIC AGENTS.}

BY ANDREW BALFOUR, M.D., B.Sc., M.R.C.P. EDIN., D.P.H. CANTAB.,

DIREOTOR, WELLCOME RESEARCH LABORATORIES, GORDON MEMORIAL COLLEGE, KHARTOUM.

IN looking over the pages of that excellent compilation the Medical Annual for 1903 I was interested to find an article stating that methylene blue has been used with success as a sedative in cases of insanity. Ehrlich and Leppmann ${ }^{1}$ in 1890 used this stain as an analgesic, administering it both internally and bypodermically, and reported favourably upon it. They were led to employ it from the fact that methylene blue will stain during life the axis cylinders of both sensory and motor nerve fibres. They stated that colouration of the nerves followed hypodermic injections. Combemale, ${ }^{2}$ Gaillard, and Piotrowski denied this action, but, on the other hand, Pilliet ${ }^{3}$ confirmed the observations of Ehrlich and Leppmann both as regards the staining of nerves and the analgesic effects, while Combemale admitted that the drug was useful in neuralgic and ataxic pains.

Some years ago I was engaged upon an investigation into the toxic properties of the dye stuffs which was embodied in an M.D. thesis, ${ }^{4}$ but the results of which I did not publish. The work dealt chiefly with the effects of these dyes on the public health and more especially with the subject of river pollution, but to some extent it considered them from a pharmacological standpoint. I experimented largely with fish-roach, dace, and gudgeon-and was surprised at some of the curious results obtained. Of peculiar interest was the apparent affinity of certain of the aniline dyes for the central nervous system. Unfortunately neither the thesis nor any of my notes are at hand and I have to rely on my memory, but I well recall that both methylene blue. tetra-methylthionine-chloride, $\mathrm{C}_{16} \mathrm{H}_{18} \mathrm{~N}_{3} \mathrm{SCl}$, and chrysoidine, the hydrochloride of diamido-azo-benzene, $\mathrm{C}_{6} \mathrm{H}_{5} \mathrm{~N}_{2} \mathrm{O}_{6} \mathrm{H}_{3}\left(\mathrm{NH}_{2}\right)_{2} \mathrm{HCl}$, exhibited this property to a marked extent. It was found that when fishes were placed even in very dilute solutions of these dyes ( 1 in 500,000 in the case of chrysoidine) they were speedily killed and on post-mortem examination were found to be stained through and through. The scales took on the stain vividly, the muscles were stained, but above all the brain and spinal cord were deeply coloured. It was hoped that this property might be utilised for staining en masse in histological work, but it was found on microscopical examination that the staining was diffuse and useless for purposes of differentiation. These two stains in particular seemed to pass very rapidly into the blood stream, no doubt vi $\hat{a}$ the gill arches, but chrysoidine was by far the most toxic. Indeed, it proved to be the most poisonous of any of the numerous aniline dyes employed. I am not aware that this dye has been used therapeutically though its disinfectant action has been tested.

Methylene blue has proved of value in malaria and to some extent also in bilharzial disease where it certainly produces sedative effects though its precise mode of action is unknown. It seems to me that chrysoidine might be given a trial.

I did not find it nearly so toxic for rabbits as for fish but it would have to be employed at first with caution and probably only after some experiments on mammals had been conducted. It has the property of precipitating the vibrios of cholera quantitatively from solutions in which they are held in suspension. The other azo compounds do not possess this property and I remember that in other ways chrysoidine was found to differ from allied chemical substances.

Any drug likely to benefit those suffering from the invasion of the Schistosoma hamatobium (to adopt the most recent nomenclature) would prove a great boon in Egypt and it is just possible that chrysoidine might succeed where others have failed, while in the light of what $I$ have stated it might also find a use in those nervous cases where methylene blue has been tried with gratifying results.

Khartoum.

1 Deutsche Medicinische Wochenschrift, June, 1902. 2 La Semaine Médicale, May, 1891.
3 La Tribune Médicale, October, 1890.

4 Andrew Balfour: The Toxic Properties of the Dye Stuffs, with special Reference to their Effects upon the Public Health and to River
Pollution (M.D. thesis, gold medal, Edinburgh, 1898). 
A CASE OF CONGENITAL DEFORMITY OF THE SKULL ASSOCIATED WITH OCULAR DEFECTS.

BY H. WORK DODD, F.R.C.S. ENG., OPHTHALMIC SURGEON TO, AND CLINICAL LECTURER ON OPHTHALMOLOGICAT MEDICINE AND SURGERY AT, THE ROYAL FREE HOSPITAL; SURGEON TO THE ROYAL WESTMLNSTER OPHTHALMIC HOSPITAL, ETC.;

AND

W. HALlibURTON MCMULLEN, B.S. LoND., F.R.C.S. ENG.,

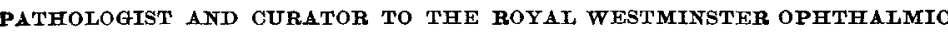
HOSPITAL; ASSISTANT OPHTHALMIC SURGEON TO THE MILLER HOSPITAL AND ROYAL KENT DISPENSARY.

THE following case is interesting both on account of the remarkable deformity of the skull and also on account of the association with this deformity of certain ocular defects.

The patient is a boy, aged seven years, who was brought to the Royal Westminster Ophthalmic Hospital on account of the prominence of his eyes and slightly defective vision. He was born at full term after a somewhat difficult labour lasting 12 hours, but no instruments were used. His father stated that the head was deformed and the eyes were prominent at birth and that the shape of the head was then much the same as it is now. This statement is confirmed by Dr. Shelmerdine of Sandgate who saw the child shortly after birth. The patient is a backward child ; he did not walk until nearly two years of age, became clean in his habits at two and $\mathbf{a}$ half years, and began to talk at three years. He has now been to school for two years but only knows a few letters and counts to four or five. For some time his parents have thought that his vision has been slightly defective. They have also noticed slight deafness which has increased lately. He has never had convulsions or fits and there is no history of rash or other signs of inherited syphilis.

FIG. 1.

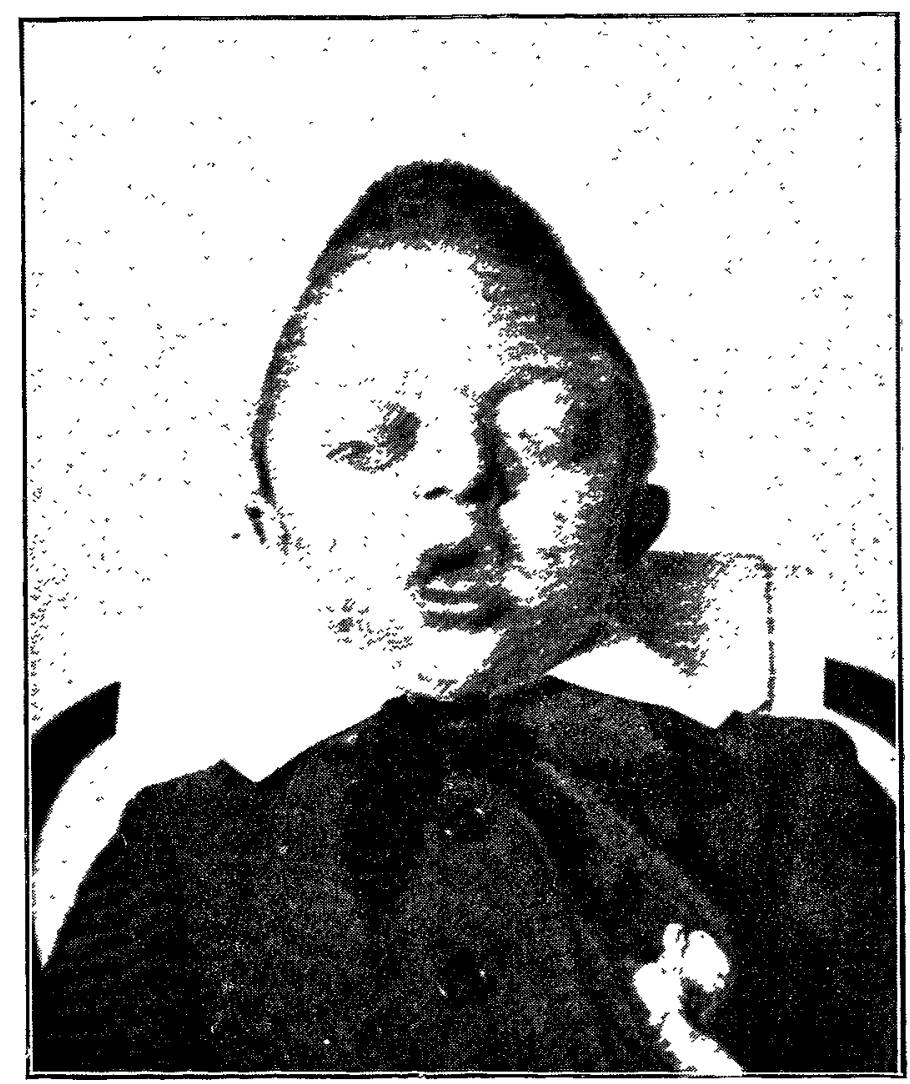

Aspect of patient, front view.

With regard to the family history the patient is the third child in a family of four. All the others are healthy, normally developed, and intelligent. There is no history of syphilis in the parents or of deformity, bone disease, insanity, or fits in any of his relatives.

The patient's present condition is as follows. Vide Figs. 1 and 2.) He is of somewhat stunted growth (height

\section{FIG. 2.}

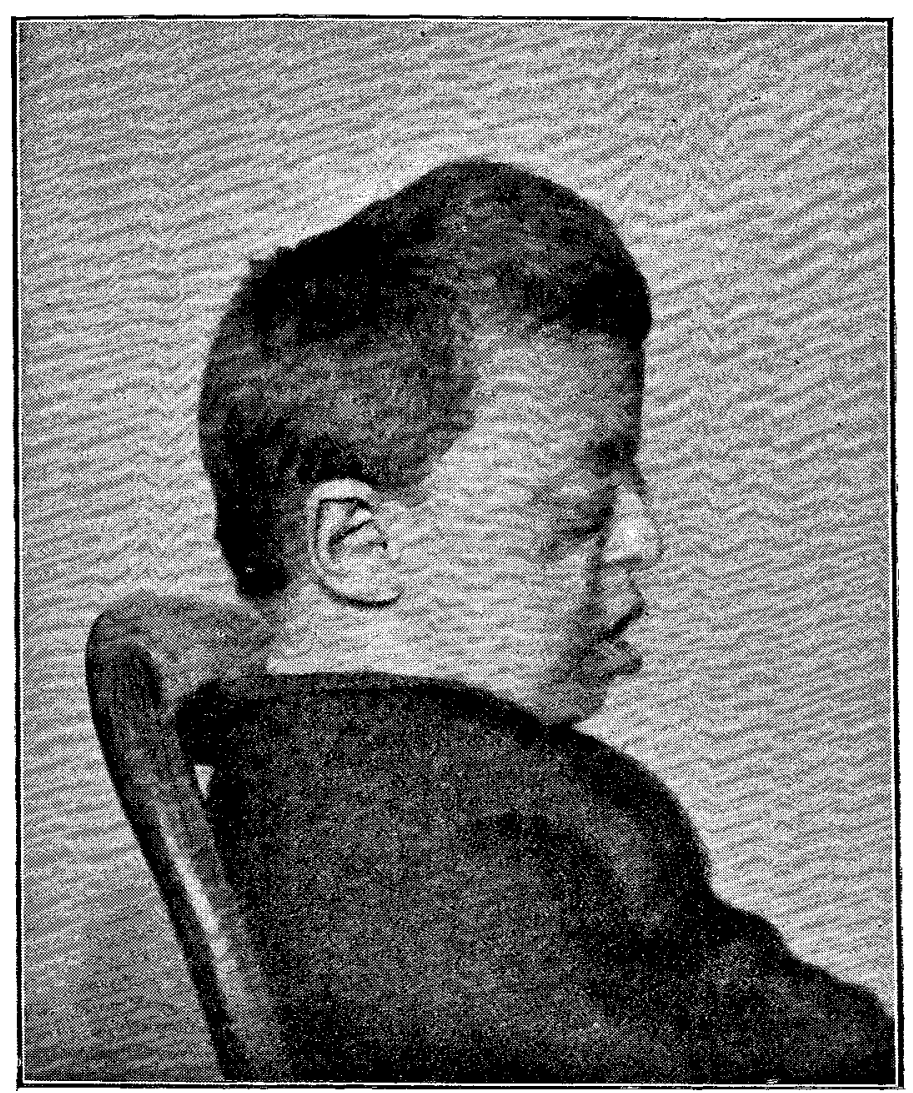

Aspect of patient, side view.

three feet eight inches) but fairly well nourished. His head is of very peculiar shape, showing remarkable projection upwards of the anterior part of the cranium and very marked bulging in the temporal regions. The face is broad and flat; the eyes are very prominent and widely separated and there are traces of epicanthic folds; the nose is small, the bridge being short and narrow; the mouth is kept open, the lips are rather thick and projecting, and the tongue protrudes slightly between them. The neck is short, not unduly thick, and there is no enlargement of the thyroid gland. His gait is clumsy and slouching and he walks with a decided stoop, the head being thrust forward. His skin is thin and supple and his hair is normal. The principal measurements of the head are as follows: horizontal circumference, 48.5 centimetres; nasooccipital arc, 36 centimetres; binauricular arc, 34 centimetres ; antero-posterior diameter, 15.6 centimetres ; greatest transverse diameter (bi-temporal), 14.2 centimetres; binauricular diameter, $11 \cdot 7$ centimetres; distance between external angular processes, 10.2 centimetres; height of face, chin to hair, $14 \cdot 5$ centimetres; height of orbits, 3.3 centimetres; and breadth of orbits, $3 \cdot 2$ centimetres. The horizontal circumference is but little below normal, the average measurement according to Still being 49.5 centimetres at"six years of age and 51.25 centimetres at eight years. $^{\text {I }}$

The most remarkable features are the great excess in height and width. The abnormal height is shown by the great relative length of the naso-occipital and binauricular ares, the former being practically equal to three-quarters of the total horizontal circumference. The great relative width is shown by the high cephalic index-i.e., $\frac{\text { breadth } \times 100}{\text {, }}$ which works out at 91.7 ; the average cephalic index in European skulls varying from 75 to 80 . The excess in height is limited to the anterior part of the cranium, being due to a very marked upward projection over the frontal region extending back only as far as a point vertically above

1 Goodhart and Still : Diseases of Children. 